$\mathrm{J}$ o u r n a l of

Mathematics

and Applications

JMA No 39, pp 69-80 (2016)

\title{
A Refinement of Schwarz's Lemma and its Applications
}

\author{
V. K. Jain
}

ABSTRACT: By using the value of the second derivative of the function at 0 , along with the values of the function and its first derivative at 0 , we have obtained a refinement of well known Schwarz's lemma and have used this refinement to obtain refinements, of Aziz and Rather's inequalities [2004] for a polynomial of degree $n$ having no zeros in $|z|<k,(k \geq 1)$.

AMS Subject Classification: Primary 30A10, Secondary 30C10.

Keywords and Phrases: Schwarz's lemma; Analytic in $|z|<1$; Polynomial; No zeros in $|z|<k, k \geq 1$, Inequalities.

\section{Introduction and statement of results}

Concerning the values of a function, analytic in the interior of a disc, we have the following well known result, known as Schwarz's lemma [2, p. 189-190].

Theorem A. If $f(z)$ is analytic in $|z|<1$, where it satisfies the inequality $|f(z)| \leq 1$, and if $f(0)=0$, then the inequality

$$
|f(z)| \leq|z|
$$

holds whenever $|z|<1$. Moreover equality can occur only when $f(z)=z e^{i \alpha}$, where $\alpha$ is a real constant.

There is a generalization of Theorem A, known as generalization of Schwarz's lemma [6, p. 212], which can be stated as

Theorem B. If $f(z)$ is analytic and $|f(z)| \leq 1$, in $|z|<1$ then

$$
|f(z)| \leq \frac{|z|+|a|}{|a||z|+1}, \quad|z|<1
$$

where

$$
a=f(0)
$$


Govil et al. [4] obtained the following refinement of Theorem B, by using the value of the first derivative of the function at 0 , along with the value of the function at 0 .

Theorem C. If $f(z)$ is analytic and $|f(z)| \leq 1$, in $|z|<1$ then

$$
|f(z)| \leq\left\{\begin{array}{ll}
\frac{(1-|a|)|z|^{2}+|b z|+|a|(1-|a|)}{|a|(1-|a|)|z|^{2}+|b z|+(1-|a|)}, & |a|<1, \\
1 & ,|a|=1,
\end{array}\right\}, \quad|z|<1,
$$

where

$$
a=f(0), b=f^{\prime}(0)
$$

The example

$$
f(z)=\left(a+\frac{b}{1+a} z-z^{2}\right) /\left(1-\frac{b}{1+a} z-a z^{2}\right)
$$

shows that the estimate is sharp.

In this paper we have firstly obtained a refinement of Theorem C, thereby giving a refinement of Theorem A also, by using the value of the second derivative of the function at 0 , along with the values of the function and its first derivative at 0 . More precisely we have proved

Theorem 1. Let $f(z)$ be analytic in $|z|<1$, with

$$
\begin{gathered}
|f(z)| \leq 1, \quad|z|<1, \\
a=f(0), \quad b=f^{\prime}(0), c=f^{\prime \prime}(0), \\
\gamma=\left\{\begin{array}{ll}
\arg \bar{a}, & a \neq 0, \\
\operatorname{any} \text { value, } & a=0,
\end{array}\right\}, \\
A=2\left(1-|a|^{2}\right)\left(1-|a|^{2}-|b|\right), \\
B=2|a||b|\left(1-|a|^{2}-|b|\right)+\left.|| a\right|^{2} c-2 e^{i \gamma}|a| b^{2}-c \mid, \\
C=2|b|\left(1-|a|^{2}-|b|\right)+\left.|a||| a\right|^{2} c-2 e^{i \gamma}|a| b^{2}-c \mid
\end{gathered}
$$

and

$$
D=2|a|\left(1-|a|^{2}\right)\left(1-|a|^{2}-|b|\right)
$$

Then

$$
|f(z)| \leq\left\{\begin{array}{ll}
\frac{A|z|^{3}+B|z|^{2}+C|z|+D}{D|z|^{3}+C|z|^{2}+B|z|+A}, & , a \mid<1 \text { and }|b|<1-|a|^{2} \\
\frac{|z|+|a|}{|a||z|+1} & ,|a|<1 \text { and }|b|=1-|a|^{2}, \\
1 & ,|a|=1
\end{array}\right\}, \quad|z|<1
$$

Remark 1. By using the result [5, p. 172, exercise \# 9] one can show that Theorem 1 is a refinement of Theorem $\mathrm{C}$. 
Further for a polynomial $p(z)$, let

$$
\begin{aligned}
\|p\|_{q} & =\left\{\frac{1}{2 \pi} \int_{0}^{2 \pi}\left|p\left(e^{i \theta}\right)\right|^{q} d \theta\right\}^{1 / q}, \quad q>0 \\
\|p\|_{\infty} & =\max _{|z|=1}|p(z)| .
\end{aligned}
$$

Then secondly we have used Theorem 1 to obtain a refinement of Aziz and Rather's result [1, Theorem 1 and Corollary 1]

Theorem D. Let $P(z)=\sum_{j=0}^{n} a_{j} z^{j}$ be a polynomial of degree $n$, having no zeros in $|z|<k,(k \geq 1)$, with

$$
1 \leq s<n
$$

and

$$
\delta_{k, s}=\frac{C(n, s)\left|a_{0}\right| k^{s+1}+\left|a_{s}\right| k^{2 s}}{C(n, s)\left|a_{0}\right|+\left|a_{s}\right| k^{s+1}} .
$$

Then

$$
\left\|P^{(s)}\right\|_{q} \leq \frac{n(n-1) \ldots(n-s+1)}{\left\|\delta_{k, s}+z\right\|_{q}}\|P\|_{q}, \quad q>0
$$

and

$\left\|P^{(s)}\right\|_{\infty} \leq n(n-1) \ldots(n-s+1)\left\{\frac{C(n, s)\left|a_{0}\right|+\left|a_{s}\right| k^{s+1}}{C(n, s)\left|a_{0}\right|\left(1+k^{s+1}\right)+k^{s+1}\left(k^{s-1}+1\right)\left|a_{s}\right|}\right\}\|P\|_{\infty}$.

More precisely we have proved

Theorem 2. Let $P(z)=\sum_{j=0}^{n} a_{j} z^{j}$ be a polynomial of degree $n$, having no zeros in $|z|<k,(k \geq 1)$, with

$$
\begin{gathered}
1 \leq s<n, \\
E=\frac{C(n, s)\left|a_{0}\right|+\left|a_{s}\right| k^{s+1}}{C(n, s)\left|a_{0}\right| k+\left|a_{s}\right| k^{s}}, \\
\delta_{k, s}=k^{s} / E,(\text { by }(1.9)), \\
F=\left|n(s+1) a_{0} a_{s+1}-(n-s) a_{1} a_{s}\right|, \\
H=\quad n\left|a_{0}\right|^{2}\{C(n, s)\}^{2}+\left|a_{0}\right|\left|a_{s}\right| n C(n, s) k^{s}\left(k^{2}-1\right)-n\left|a_{s}\right|^{2} k^{2 s+2} \\
+k^{s+2} C(n, s) F, \\
n k^{2}\{C(n, s)\}^{2}\left|a_{0}\right|^{2}-n C(n, s)\left|a_{0}\right|\left|a_{s}\right| k^{s}\left(k^{2}-1\right)-n\left|a_{s}\right|^{2} k^{2 s} \\
+k^{s+2} C(n, s) F,
\end{gathered}
$$




$$
\begin{aligned}
& d_{k, s}=\left\{\begin{array}{l}
k^{s} J / H, \frac{k^{s}}{C(n, s)}\left|\frac{a_{s}}{a_{0}}\right|<1, \\
k^{s}, \frac{k^{s}}{C(n, s)}\left|\frac{a_{s}}{a_{0}}\right|=1,
\end{array}\right. \\
& a_{n+1}=0, \\
& \gamma=\left\{\begin{array}{l}
\arg \left(\frac{k^{s} \overline{a_{s}}}{C(n, s) \overline{a_{0}}}\right), \frac{k^{s} a_{s}}{C(n, s) a_{0}} \neq 0, \frac{k^{s} a_{s}}{C(n, s) a_{0}}=0 \\
\text { any value }
\end{array}\right. \\
& G=\mid a_{0}\left\{n^{2}(n-1) a_{0}^{2}(s+2)(s+1) a_{s+2}-2 n(n-s) a_{0}\left((n-s-1) a_{2} a_{s}+\right.\right. \\
& \left.\left.(n-1)(s+1) a_{1} a_{s+1}\right)+2(n-1)(n-s)^{2} a_{1}^{2} a_{s}\right\}\left\{k^{2 s}\left|a_{s}\right|^{2}-\right. \\
& \left.(C(n, s))^{2}\left|a_{0}\right|^{2}\right\}-2 e^{i \gamma}(n-1)\left|a_{0}\right|\left|a_{s}\right| k^{2 s}\left\{n(s+1) a_{0} a_{s+1}-\right. \\
& \left.(n-s) a_{1} a_{s}\right\}^{2} \mid \text {, } \\
& L=2 n^{2}(n-1)\left\{(C(n, s))^{5}\left|a_{0}\right|^{7}+(C(n, s))^{4} k^{s+3}\left|a_{0}\right|^{6}\left|a_{s}\right|-\right. \\
& 2(C(n, s))^{3} k^{2 s}\left|a_{0}\right|^{5}\left|a_{s}\right|^{2}-2(C(n, s))^{2} k^{3 s+3}\left|a_{0}\right|^{4}\left|a_{s}\right|^{3} \\
& \left.+C(n, s) k^{4 s}\left|a_{0}\right|^{3}\left|a_{s}\right|^{4}+k^{5 s+3}\left|a_{0}\right|^{2}\left|a_{s}\right|^{5}\right\}+ \\
& 2 n(n-1) C(n, s) k^{s+1}\left|a_{0}\right|^{2} F\left(k^{2}-1\right)\left\{\left|a_{0}\right|^{3}(C(n, s))^{3}-\right. \\
& (C(n, s))^{2}\left|a_{0}\right|^{2}\left|a_{s}\right| k^{s+1}-(C(n, s))\left|a_{0}\right|\left|a_{s}\right|^{2} k^{2 s}+ \\
& \left.\left|a_{s}\right|{ }^{3} k^{3 s+1}\right\}+C(n, s) k^{s+3} G\left\{C(n, s)\left|a_{0}\right|+\left|a_{s}\right| k^{s+1}\right\}- \\
& 2(n-1)(C(n, s))^{2} k^{2 s+3} F^{2}\left|a_{0}\right|^{2}\left\{k C(n, s)\left|a_{0}\right|+k^{s}\left|a_{s}\right|\right\}, \\
& S=2 n^{2}(n-1)\left|a_{0}\right|^{2}\left\{(C(n, s))^{5}\left|a_{0}\right|^{5} k^{3}+(C(n, s))^{4}\left|a_{0}\right|^{4}\left|a_{s}\right| k^{s}-\right. \\
& 2(C(n, s))^{3}\left|a_{0}\right|^{3}\left|a_{s}\right|^{2} k^{2 s+3}-2(C(n, s))^{2}\left|a_{0}\right|^{2}\left|a_{s}\right|^{3} k^{3 s}+ \\
& \left.C(n, s)\left|a_{0}\right|\left|a_{s}\right|{ }^{4} k^{4 s+3}+\left|a_{s}\right|{ }^{5} k^{5 s}\right\}+2 n(n-1)\left|a_{0}\right|^{2} k^{s+1} C(n, s)\left(k^{2}-1\right) \\
& F\left\{-(C(n, s))^{3} k\left|a_{0}\right|^{3}+(C(n, s))^{2} k^{s}\left|a_{0}\right|^{2}\left|a_{s}\right|+C(n, s) k^{2 s+1}\left|a_{0}\right|\left|a_{s}\right|^{2}\right. \\
& \left.-\left|a_{s}\right|^{3} k^{3 s}\right\}-2(n-1)(C(n, s))^{2} k^{2 s+3}\left|a_{0}\right|^{2} F^{2}\left\{\left|a_{0}\right| C(n, s)+\right. \\
& \left.k^{s+1}\left|a_{s}\right|\right\}+G k^{s+3} C(n, s)\left\{\left|a_{0}\right| k+\left|a_{s}\right| k^{s}\right\} \text {, } \\
& \gamma_{k, s}=\left\{\begin{array}{l}
\frac{k^{s} S}{L}, \frac{k^{s}}{C(n, s)}\left|\frac{a_{s}}{a_{0}}\right|<1 \text { and } \frac{k^{s+1} F}{n\left|a_{0}\right|^{2} C(n, s)}<1-\frac{k^{2 s}}{(C(n, s))^{2}}\left|\frac{a_{s}}{a_{0}}\right|^{2}, \\
\frac{k^{s}}{E}, \frac{k^{s}}{C(n, s)}\left|\frac{a_{s}}{a_{0}}\right|<1 \text { and } \frac{k^{s+1} F}{n\left|a_{0}\right|^{2} C(n, s)}=1-\frac{k^{s}}{(C(n, s))^{2}}\left|\frac{a_{s}}{a_{0}}\right|^{2}, \\
k^{s}, \frac{k^{s}}{C(n, s)}\left|\frac{\mid a_{s}}{a_{0}}\right|=1 .
\end{array}\right.
\end{aligned}
$$


Then for $q>0$

$$
\begin{aligned}
\left\|P^{(s)}\right\|_{q} & \leq \frac{n(n-1) \ldots(n-s+1)}{\left\|\gamma_{k, s}+z\right\|_{q}}\|P\|_{q}, \\
& \leq \frac{n(n-1) \ldots(n-s+1)}{\left\|d_{k, s}+z\right\|_{q}}\|P\|_{q} \\
& \leq \frac{n(n-1) \ldots(n-s+1)}{\left\|\delta_{k, s}+z\right\|_{q}}\|P\|_{q} \\
& \leq \frac{n(n-1) \ldots(n-s+1)}{\left\|k^{s}+z\right\|_{q}}\|P\|_{q} .
\end{aligned}
$$

Remark 2. With the additional assumption

$$
a_{n+2}=0,
$$

one can show that Theorem 2 is true for $s=n$ also.

By letting $q \rightarrow \infty$ in (1.18), we obtain

Corollary 1. Under the same hypotheses as in Theorem 2

$$
\begin{aligned}
\left\|P^{(s)}\right\|_{\infty} & \leq \frac{n(n-1) \ldots(n-s+1)}{\left(\gamma_{k, s}+1\right)}\|P\|_{\infty}, \\
& \leq \frac{n(n-1) \ldots(n-s+1)}{\left(d_{k, s}+1\right)}\|P\|_{\infty}, \\
& \leq \frac{n(n-1) \ldots(n-s+1)}{\left(\delta_{k, s}+1\right)}\|P\|_{\infty}, \\
& \leq \frac{n(n-1) \ldots(n-s+1)}{\left(k^{s}+1\right)}\|P\|_{\infty} .
\end{aligned}
$$

Remark 3. With the additional assumption

$$
a_{n+2}=0,
$$

one can show that Corollary 1 is true for $s=n$ also.

Remark 4. Corollary 1 is also a refinement of Govil and Rahman's result [3, Theorem 4].

\section{Lemmas}

For the proofs of the theorems we require the following lemmas.

Lemma 1. If $f(z)$ is analytic and $|f(z)| \leq 1$, in $|z| \leq 1$ then

$$
|f(z)| \leq \frac{|z|+|a|}{1+|a||z|}, \quad|z| \leq 1,
$$

where

$$
a=f(0)
$$


Proof of Lemma 1. It easily follows from Theorem B.

Lemma 2. If $f(z)$ is analytic and $|f(z)| \leq 1$, in $|z| \leq 1$ then

$$
|f(z)| \leq\left\{\begin{array}{ll}
\frac{(1-|a|)|z|^{2}+|b z|+|a|(1-|a|)}{|a|(1-|a|)|z|^{2}+|b z|+(1-|a|)}, & ,|a|<1, \\
1 & ,|a|=1,
\end{array}\right\}, \quad|z| \leq 1,
$$

where

$$
a=f(0), b=f^{\prime}(0) .
$$

Proof of Lemma 2. It easily follows from Theorem C.

Remark 5. By using the result [5, p.172, exercise \# 9] one can show that Lemma 2 is a refinement of Lemma 1.

Lemma 3. Let $f(z)$ be analytic in $|z| \leq 1$, with

$$
\begin{gathered}
|f(z)| \leq 1, \quad|z| \leq 1, \\
a=f(0), b=f^{\prime}(0), c=f^{\prime \prime}(0), \\
\gamma=\left\{\begin{array}{ll}
\arg \bar{a} & , a \neq 0, \\
\text { any value }, & a=0,
\end{array}\right\} .
\end{gathered}
$$

Then

$$
|f(z)| \leq\left\{\begin{array}{ll}
\frac{A|z|^{3}+B|z|^{2}+C|z|+D}{D D||^{3}+C|z|^{2}+B|z|+A}, & |a|<1 \text { and }|b|<1-|a|^{2}, \\
\frac{|z|+|a|}{1+|a||z|} & ,|a|<1 \text { and }|b|=1-|a|^{2}, \\
1 & ,|a|=1
\end{array}\right\}, \quad|z| \leq 1
$$

where $A, B, C$ and $D$ are, as in Theorem 1.

Proof of Lemma 3. It easily follows from Theorem 1.

Remark 6. By Remark 1 one can say that Lemma 3 is a refinement of Lemma 2.

Lemma 4. Under the same hypotheses as in Theorem 2

$$
k^{s}\left|P^{(s)}(z)\right| \leq \delta_{k, s}\left|P^{(s)}(z)\right| \leq d_{k, s}\left|P^{(s)}(z)\right| \leq \gamma_{k, s}\left|P^{(s)}(z)\right| \leq\left|Q^{(s)}(z)\right|,|z|=1,
$$

where

$$
Q(z)=z^{n} \overline{P(1 / \bar{z})}
$$

Proof of Lemma 4. Let

$$
\begin{aligned}
G(z) & =z^{n} \overline{P(k / \bar{z})}=k^{n} Q(z / k), \\
H(z) & =z^{n-s} \overline{G^{(s)}(1 / \bar{z})}=(k z)^{n-s} \overline{Q^{(s)}(1 /(k \bar{z}))} .
\end{aligned}
$$


Then by using the result [1, inequality 32$]$ we get

$$
k^{s}\left|P^{(s)}(k z)\right| \leq|H(z)|, \quad|z|=1,
$$

with $H(z)$ having all its zeros in $|z| \geq 1$. Further let

$$
H(z)=\Phi(z) H_{1}(z)
$$

with

$$
H_{1}(z) \neq 0, \quad|z|=1
$$

and

$$
\Phi(z)= \begin{cases}1 & ; H(z) \neq 0 \text { on }|z|=1 \\ \Pi_{\gamma=1}^{m}\left(z-z_{\gamma}\right),\left|z_{\gamma}\right|=1 \forall \gamma ; H(z) \text { has certain zeros on }|z|=1 .\end{cases}
$$

Then by (2.4), (2.5) and (2.6) we have

$$
k^{s} P^{(s)}(k z)=\Phi(z) P_{1}(z),
$$

with

$$
\left|P_{1}(z)\right| \leq\left|H_{1}(z)\right|, \quad|z|=1 .
$$

Now

$$
f(z)=\frac{P_{1}(z)}{H_{1}(z)}
$$

is analytic in $|z| \leq 1$, with

$$
|f(z)| \leq 1, \quad|z|=1, \quad(\text { by }(2.8))
$$

and therefore

$$
|f(z)| \leq 1, \quad|z| \leq 1, \text { (by maxiumum modulus principle) }
$$

with

$$
\begin{gathered}
|f(z)| \leq \frac{|z|+|a|}{1+|a||z|}, \quad|z| \leq 1, \quad(\text { a refinement of }(2.10)), \\
|f(z)| \leq\left\{\begin{array}{ll}
\frac{(1-|a|)|z|^{2}+|b||z|+|a|(1-|a|)}{|a|(1-|a|)|z|^{2}+|b||z|+(1-|a|)}, & |a|<1, \\
1 & ,|a|=1,
\end{array}\right\}, \quad|z| \leq 1,
\end{gathered}
$$

(a refinement of (2.11)), (by Remark 5)

and

$$
|f(z)| \leq\left\{\begin{array}{ll}
\frac{A|z|^{3}+B|z|^{2}+C|z|+D}{D|z|^{3}+C|z|^{2}+B|z|+A} & ,|a|<1 \text { and }|b|<1-|a|^{2} \\
\frac{|z|+|a|}{1+|a||z|} & ,|a|<1 \text { and }|b|=1-|a|^{2}, \\
1 & ,|a|=1
\end{array}\right\}, \quad|z| \leq 1
$$

(a refinement of (2.12)), (by Remark 6). 
Further if

$$
g(z)=\frac{k^{s} P^{(s)}(k z)}{H(z)}
$$

then

$$
\begin{gathered}
a=f(0),(\text { by }(1.2)), \\
=g(0),(\text { by }(2.5),(2.7) \text { and }(2.9)), \\
=\frac{k^{s} a_{s}}{C(n, s) a_{0}}, \\
b=f^{\prime}(0),(\text { by }(1.2)), \\
=g^{\prime}(0),(\text { by }(2.5),(2.7) \text { and }(2.9)), \\
=\frac{k^{s+1}}{n a_{0}^{2} C(n, s)}\left\{n(s+1) a_{0} a_{s+1}-(n-s) a_{1} a_{s}\right\}, \\
=\quad f^{\prime \prime}(0),(\text { by }(1.2)), \\
=\quad g^{\prime \prime}(0),(\text { by }(2.5),(2.7) \text { and }(2.9)), \\
=\frac{k^{s+2}}{n^{2}(n-1) a_{0}^{3} C(n, s)}\left\{n^{2}(n-1) a_{0}^{2}(s+1)(s+2) a_{s+2}-\right. \\
2 n(n-s) a_{0}\left((n-s-1) a_{2} a_{s}+\right. \\
\left.\left.(n-1)(s+1) a_{1} a_{s+1}\right)+2(n-1)(n-s)^{2} a_{1}^{2} a_{s}\right\}
\end{gathered}
$$

and on using (2.13), with

$$
z=\frac{1}{k} e^{i \theta}, \quad 0 \leq \theta \leq 2 \pi
$$

we get

$$
\left|f\left(\frac{1}{k} e^{i \theta}\right)\right| \leq\left\{\begin{array}{l}
L / S, \frac{k^{s}}{C(n, s)}\left|\frac{a_{s}}{a_{0}}\right|<1 \text { and } \frac{k^{s+1} F}{n\left|a_{0}\right|^{2} C(n, s)}<1-\frac{k^{2 s}}{(C(n, s))^{2}}\left|\frac{a_{s}}{a_{0}}\right|^{2}, \\
E \quad, \frac{k^{s}}{C(n, s)}\left|\frac{a_{s}}{a_{0}}\right|<1 \text { and } \frac{k^{s+1} F}{n\left|a_{0}\right|^{2} C(n, s)}=1-\frac{k^{2 s}}{(C(n, s))^{2}}\left|\frac{a_{s}}{a_{0}}\right|^{2}, \\
1 \quad, \frac{k^{s}}{C(n, s)}\left|\frac{a_{s}}{a_{0}}\right|=1,
\end{array}\right\}
$$

(by $(2.14),(2.15),(2.16),(1.3),(1.4),(1.5),(1.6),(1.7),(1.11),(1.12),(1.13),(1.14)$ and $(1.10))$,

i.e.

$\left|P_{1}\left(\frac{1}{k} e^{i \theta}\right)\right| \leq\left\{\begin{array}{l}L / S, \frac{k^{s}}{C(n, s)}\left|\frac{a_{s}}{a_{0}}\right|<1 \text { and } \frac{k^{s+1} F}{n\left|a_{0}\right|^{2} C(n, s)}<1-\frac{k^{2 s}}{(C(n, s))^{2}}\left|\frac{a_{s}}{a_{0}}\right|^{2}, \\ E, \frac{k^{s}}{C(n, s)}\left|\frac{a_{s}}{a_{0}}\right|<1 \text { and } \frac{k^{s+1} F}{n\left|a_{0}\right|^{2} C(n, s)}=1-\frac{k^{2 s}}{(C(n, s))^{2}}\left|\frac{a_{s}}{a_{0}}\right|^{2}, \\ 1, \frac{k^{s}}{C(n, s)}\left|\frac{a_{s}}{a_{0}}\right|=1,\end{array}\right\}\left|H_{1}\left(\frac{1}{k} e^{i \theta}\right)\right|$,

(by (2.9)), 
which, by (2.5) and (2.7), implies that

$$
\begin{aligned}
& k^{s}\left|P^{(s)}\left(e^{i \theta}\right)\right| \\
& \quad \leq\left\{\begin{array}{l}
L / S, \frac{k^{s}}{C(n, s)}\left|\frac{a_{s}}{a_{0}}\right|<1 \text { and } \frac{k^{s+1} F}{n\left|a_{0}\right|^{2} C(n, s)}<1-\frac{k^{2 s}}{(C(n, s))^{2}}\left|\frac{a_{s}}{a_{0}}\right|^{2}, \\
E, \frac{k^{s}}{C(n, s)}\left|\frac{a_{s}}{a_{0}}\right|<1 \text { and } \frac{k^{s+1} F}{n\left|a_{0}\right|^{2} C(n, s)}=1-\frac{k^{2 s}}{(C(n, s))^{2}}\left|\frac{a_{s}}{a_{0}}\right|^{2}, \\
1, \frac{k^{s}}{C(n, s)}\left|\frac{a_{s}}{a_{0}}\right|=1,
\end{array}\right\}\left|H\left(\frac{1}{k} e^{i \theta}\right)\right| .
\end{aligned}
$$

And as

$$
H(z / k)=z^{n-s} \overline{Q^{(s)}(1 / \bar{z})}, \quad(\text { by }(2.3)),
$$

we get, by using $(1.15),(1.16)$ and (1.17) in (2.17), that

$$
\gamma_{k, s}\left|P^{(s)}\left(e^{i \theta}\right)\right| \leq\left|Q^{(s)}\left(e^{i \theta}\right)\right|, \quad 0 \leq \theta \leq 2 \pi .
$$

Now as we have obtained (2.18) by using (2.13), we can similarly obtain

$$
\begin{aligned}
& d_{k, s}\left|P^{(s)}\left(e^{i \theta}\right)\right| \leq\left|Q^{(s)}\left(e^{i \theta}\right)\right|, \quad 0 \leq \theta \leq 2 \pi, \\
& \delta_{k, s}\left|P^{(s)}\left(e^{i \theta}\right)\right| \leq\left|Q^{(s)}\left(e^{i \theta}\right)\right|, \quad 0 \leq \theta \leq 2 \pi
\end{aligned}
$$

and

$$
k^{s}\left|P^{(s)}\left(e^{i \theta}\right)\right| \leq\left|Q^{(s)}\left(e^{i \theta}\right)\right|, \quad 0 \leq \theta \leq 2 \pi,
$$

by using (2.12), (2.11) and (2.10) respectively. Further

$$
k^{s} \leq \delta_{k, s} \leq d_{k, s} \leq \gamma_{k, s}
$$

follows from the fact

$$
\begin{gathered}
\left\{\begin{aligned}
\frac{A|z|^{3}+B|z|^{2}+C|z|+D}{D|z|^{3}+C|z|^{2}+B|z|+A} & ,|a|<1 \text { and }|b|<1-|a|^{2} \\
\frac{|z|+|a|}{1+|a||z|} & ,|a|<1 \text { and }|b|=1-|a|^{2} \\
1 & ,|a|=1,
\end{aligned}\right\} \\
\leq\left\{\begin{array}{cc}
\frac{(1-|a|)|z|^{2}+|b||z|+|a|(1-|a|)}{|a|(1-|a|)|z|^{2}+|b||z|+(1-|a|)} & ,|a|<1 \\
1 & ,|a|=1,
\end{array}\right\} \leq \frac{|z|+|a|}{1+|a||z|} \leq 1, \quad|z| \leq 1 \\
\text { (by (2.13), (2.12), (2.11) and (2.10)) }
\end{gathered}
$$

and the way, L. H. S. of inequality (2.17) was obtained from R. H. S. of inequality (2.18). This completes the proof of Lemma 4.

Remark 7. With the additional assumption

$$
a_{n+2}=0,
$$

one can show that Lemma 4 is true for $s=n$ also. 


\section{Proofs of the theorems}

Proof of Theorem 1. If

$$
|f(0)|=1
$$

then result follows trivially. Therefore from now onwards we will assume that

$$
|f(0)|<1
$$

Now we consider the function

$$
g(z)=\frac{e^{i \gamma} f(z)-|f(0)|}{|f(0)| e^{i \gamma} f(z)-1}
$$

which is analytic in $|z|<1$, with

$$
\begin{aligned}
|g(z)| & \leq 1, \quad|z|<1 \\
g(0) & =0, \\
|g(z)| & \leq|z|, \quad|z|<1, \quad \text { by Schwarz's lemma), } \\
g^{\prime}(0) & =\frac{e^{i \gamma} f^{\prime}(0)}{|f(0)|^{2}-1} \\
g^{\prime \prime}(0) & =\frac{e^{i \gamma}}{\left(|f(0)|^{2}-1\right)^{2}}\left[f^{\prime \prime}(0)\left\{|f(0)|^{2}-1\right\}-2 e^{i \gamma}|f(0)|\left(f^{\prime}(0)\right)^{2}\right]
\end{aligned}
$$

and the function

$$
\Phi(z)= \begin{cases}\frac{g(z)}{z}, & 0<|z|<1 \\ g^{\prime}(0), & z=0\end{cases}
$$

which is analytic in $|z|<1$, with

$$
\begin{aligned}
|\Phi(z)| & \leq 1, \quad|z|<1 \\
\Phi^{\prime}(0) & =\frac{1}{2} g^{\prime \prime}(0) .
\end{aligned}
$$

We apply Theorem $\mathrm{C}$ to $\Phi(z)$. If

$$
|\Phi(0)|=1
$$

then by (1.1) and (3.4)

$$
\left|\frac{g(z)}{z}\right| \leq 1, \quad 0<|z|<1
$$

i.e.

$$
\left.\left|\frac{e^{i \gamma} f(z)-|f(0)|}{|f(0)| e^{i \gamma} f(z)-1}\right| \leq|z|, \quad|z|<1, \quad \text { by }(3.1)\right) \text {, }
$$


which implies

$$
\left|e^{i \gamma} f(z)\right| \leq \frac{|z|+|f(0)|}{1+|f(0)||z|}, \quad|z|<1
$$

thereby proving second part of (1.8). And if

$$
|\Phi(0)|<1
$$

then by (1.1) and (3.4)

$$
\left|\frac{g(z)}{z}\right| \leq \frac{(1-|\Phi(0)|)|z|^{2}+\left|\Phi^{\prime}(0)\right||z|+|\Phi(0)|(1-|\Phi(0)|)}{|\Phi(0)|(1-|\Phi(0)|)|z|^{2}+\left|\Phi^{\prime}(0)\right||z|+(1-|\Phi(0)|)}, \quad 0<|z|<1
$$

i.e.

$$
\begin{aligned}
\left|\frac{e^{i \gamma} f(z)-|f(0)|}{|f(0)| e^{i \gamma} f(z)-1}\right| & \leq|z| \frac{(1-|\Phi(0)|)|z|^{2}+\left|\Phi^{\prime}(0)\right||z|+|\Phi(0)|(1-|\Phi(0)|)}{|\Phi(0)|(1-|\Phi(0)|)|z|^{2}+\left|\Phi^{\prime}(0)\right||z|+(1-|\Phi(0)|)},|z|<1, \quad(\operatorname{by}(3.1)), \\
& =E_{0}, \text { (say), }|z|<1,
\end{aligned}
$$

which implies

$$
\begin{aligned}
\left|e^{i \gamma} f(z)\right| \leq & \frac{E_{0}+|f(0)|}{1+E_{0}|f(0)|}, \quad|z|<1 \\
= & \frac{A|z|^{3}+B|z|^{2}+C|z|+D}{D|z|^{3}+C|z|^{2}+B|z|+A}, \quad|z|<1, \\
& (\text { by }(3.5),(3.6),(3.2),(3.3),(1.2),(1.4),(1.5),(1.6) \text { and }(1.7)),
\end{aligned}
$$

thereby proving first part of (1.8). This completes the proof of Theorem 1 .

Proof of Theorem 2. As Aziz and Rather [1, Theorem 1] had obtained

$$
\left\|P^{(s)}\right\|_{q} \leq \frac{n(n-1) \ldots(n-s+1)}{\left\|\delta_{k, s}+z\right\|_{q}}\|P\|_{q},
$$

by using

$$
\delta_{k, s}\left|P^{(s)}(z)\right| \leq\left|Q^{(s)}(z)\right|, \quad|z|=1, \quad(\text { by }(2.2) \text { and }(2.1))
$$

one can similarly obtain

$$
\begin{aligned}
\left\|P^{(s)}\right\|_{q} & \leq \frac{n(n-1) \ldots(n-s+1)}{\left\|\gamma_{k, s}+z\right\|_{q}}\|P\|_{q}, \\
\left\|P^{(s)}\right\|_{q} & \leq \frac{n(n-1) \ldots(n-s+1)}{\left\|d_{k, s}+z\right\|_{q}}\|P\|_{q}
\end{aligned}
$$


and

by using

$$
\left\|P^{(s)}\right\|_{q} \leq \frac{n(n-1) \ldots(n-s+1)}{\left\|k^{s}+z\right\|_{q}}\|P\|_{q},
$$

$$
\begin{aligned}
& \left.\gamma_{k, s}\left|P^{(s)}(z)\right| \leq\left|Q^{(s)}(z)\right|, \quad|z|=1, \quad \text { by }(2.2) \text { and }(2.1)\right) \\
& \left.d_{k, s}\left|P^{(s)}(z)\right| \leq\left|Q^{(s)}(z)\right|, \quad|z|=1, \quad \text { by }(2.2) \text { and }(2.1)\right)
\end{aligned}
$$

and

$$
\left.k^{s}\left|P^{(s)}(z)\right| \leq\left|Q^{(s)}(z)\right|, \quad|z|=1 \text {, (by (2.2) and }(2.1)\right)
$$

respectively. Further

$$
1 \leq k^{s} \leq \delta_{k, s} \leq d_{k, s} \leq \gamma_{k, s}, \quad(\text { by }(2.19))
$$

implies

$$
\left|k^{s}+e^{i \theta}\right| \leq\left|\delta_{k, s}+e^{i \theta}\right| \leq\left|d_{k, s}+e^{i \theta}\right| \leq\left|\gamma_{k, s}+e^{i \theta}\right|, \quad 0 \leq \theta \leq 2 \pi,
$$

thereby proving last three inequalities in (1.18). This completes the proof of Theorem 2.

\section{References}

[1] A. Aziz, N.A. Rather, Some Zygmund type $L^{q}$ inequalities for polynomials, J. Math. Anal. Appl. 289 (2004) 14-29.

[2] E.T. Copson, An Introduction to the Theory of Functions of a Complex Variable, The English Language Book Society and Oxford University Press, Oxford, 1961.

[3] N.K. Govil, Q.I. Rahman, Functions of exponential type not vanishing in a half plane and related polynomials, Trans. Amer. Math. Soc. 137 (1969) 501-517.

[4] N.K. Govil, Q.I. Rahman, G. Schmeisser, On the derivative of a polynomial, Ill. J. Maths. 23 (1979) 319-329.

[5] Z. Nehari, Conformal Mapping, $1^{\text {st }}$ edition, McGraw-Hill, New York, 1952.

[6] E.C. Titchmarsh, The Theory of Functions, The English Language Book Society and Oxford University Press, London, 1962.

\section{DOI: $10.7862 /$ rf.2016.5}

\section{K. Jain}

email: vinayjain.kgp@gmail.com

Mathematics Department, I.I.T.,

Kharagpur - 721302, India.

Received 15.02.2015

Accepted 20.05.2016 